\title{
Photoelectrochemical Photocurrent Switching Effect: A New Platform for Molecular Logic Devices
}

\author{
Konrad Szaciłowski and Wojciech Macyk \\ Dedicated to Professor Horst Kisch on the occasion of his $65^{\text {th }}$ birthday
}

\begin{abstract}
Unusual photoelectrochemical properties of titanium dioxide modified with iron(II) complexes allow application of these materials for the construction of various optoelectronic devices including switches, logic gates and even demultiplexers. All these devices use optical input and electric output. The mechanism of the photoelectrochemical photocurrent switching (PEPS) effect is described in detail on the basis of experimental data and quantum-chemical calculations. All the collected data indicate the importance of the complex-semiconductor binding mode.
\end{abstract}

Keywords: Ferrocene · Hexacyanoferrate · PEPS effect · Photoelectrochemistry · Titanium dioxide

Increasing demand for computational power forces very rapid development of semiconductor-based electronic devices. The efficiency of these devices is strongly correlated with the size of elemental components: the smaller they are the faster they can operate. Miniaturization of classical electronic devices is limited by physical, material and technological limits. ${ }^{[1]}$

Application of various molecular, supramolecular and nanoscopic entities for digital information processing seems very promising. In some opinions application of molecular structures for information storage and processing may bypass, at least temporarily, the limitations of classical semiconductor devices and sustain the development of information technologies, ${ }^{[2-15]}$ although there are still issues that need to be solved.

${ }^{\star}$ Correspondence: Dr. K. Szaciłowski Centre for Inorganic Nanochemistry nanolnchem Faculty of Chemistry

Jagiellonian University

ul. Romana Ingardena 3, 30-060 Kraków, Poland

E-Mail: szacilow@chemia.uj.edu.pl
There are thousands of molecular logic devices. In most cases they use chemical stimuli as inputs and changes in optical properties (absorbance, fluorescence) as outputs. This approach makes the interfacing of molecular devices with macroscopic world extremely difficult. The same difficulties are met on concatenation of individual molecular devices.

Nowadays most of the research efforts are concentrated on organic molecular devices, ${ }^{[16-23]}$ with special emphasis to self-assembly of supramolecular structures.[24-26] It seems, however, that inorganic and hybrid nanocrystalline materials may be used for construction of functional information processing devices as well. Therefore detailed research on the application of wide band gap semiconductors was undertaken in order to develop new molecular basis for logic devices which operate under conditions related to classical electronic elements.

Surface modified wide band gap semiconductors and nanocomposites incorporating semiconductor particles and coordination or organometallic compounds are novel materials that show numerous extraordinary properties. Various synthetic methods are applied in preparation of semiconductor nanocomposites, including electrodeposition, wet impregnation, suspension polymerization, mechanical mixing, chemical vapor deposition, sputtering and others. ${ }^{[27]}$ Nanocomposite materials usually show improvement of performance as compared to neat constituents concerning such properties as stability, (photo)catalytic activity, efficiency of solar energy conversion and others. Semiconductor nanoparticles usually serve as light harvesting antennae or (photo)catalytic centers, while matrices, apart from purely mechanical support, may function as electron buffers, photosensitizers, protecting layers etc.

Various iron(II) complexes are very suitable modifiers for nanocrystalline $\mathrm{TiO}_{2}$ surfaces. Cyanoferrates can be very easily immobilized onto titanium dioxide surfaces owing to formation of the stable $\mathrm{Ti}-\mathrm{N} \equiv \mathrm{C}-\mathrm{Fe}$ framework. This type of bonding constitutes a good platform for a strong electronic coupling between surface species and electronic continuum of a semiconductor. ${ }^{[28,29]}$ Alternatively, redox active complexes can be immobilized via ligands equipped with anchoring groups (carboxylic, phosphonic, boronic, etc.). Such a solution is very often used for instance in the construction of dyesensitized solar cells - this mode of binding supports a much weaker electronic interaction between the molecule and the semiconducting substrate. The weakest interaction can be observed in the case of physisorption of molecules onto $\mathrm{TiO}_{2}$ surfaces.[30] The strength of electronic coupling can be easily deduced from diffuse reflectance spectra of modified semiconductors.

Chemisorption of selected $\mathrm{Fe}^{\mathrm{II}}$ species onto $\mathrm{TiO}_{2}$ surface yields materials with 


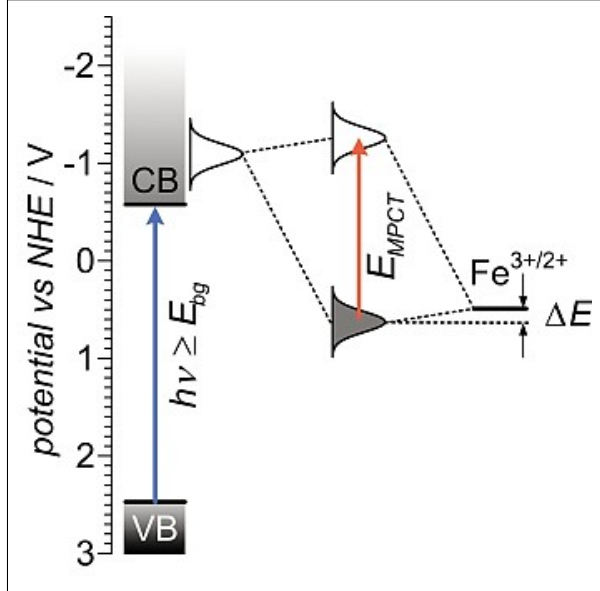

Fig. 1. The simplified energy diagram for the cyanoferrate-titanium dioxide system

new spectral features. Composite materials show characteristic absorption associated with an indirect band gap and transition associated with the surface molecules themselves (e.g. MLCT, $\pi-\pi^{*}$, etc.). In the case of a strong electronic coupling between the surface species and electronic continuum of semiconductor bands (especially the unoccupied surface states) new bands of the MPCT (metal-to-particle charge transfer) character are observed (Fig. 1). The intensity and width of these bands are a measure of electronic coupling between the surface species and semiconductor. ${ }^{[30,31]}$ This interaction also results in an increase of the surface species redox potential (Fig. 1). In the case of pentacyanoferrates the redox potential shift depends on the nucleophilicity of the axial ligand and varies from $5 \mathrm{mV}$ (2,2'-thiodiacetate) to $193 \mathrm{mV}$ (methionine). ${ }^{[32]}$

Upon excitation neat titanium dioxide generates anodic photocurrents (Fig. 2a). Immobilization of redox active species (e.g. penta- ${ }^{[32]}$ or hexacyanoferrates, ${ }^{[33]}$ Prussian blue, ${ }^{[34]}$ ferrocene and its derivatives ${ }^{[30]}$ ) onto its surface yields materials which upon optical stimulation can generate cathodic or anodic photocurrents depending on the photoelectrode potential, wavelength and electrolyte composition. ${ }^{[30,32-37]}$ It was found that in the case of iron complexes only the reduced form $\left(\mathrm{Fe}^{\mathrm{II}}\right)$ can contribute to cathodic photocurrent generation, while upon the surface complex oxidation materials behave like untreated $\mathrm{TiO}_{2}$. Excitation of the photoelectrodes made of a modified semiconductor within the MPCT bands results in most cases in generation of cathodic photocurrent, while a direct excitation of the semiconductor results in cathodic or anodic photocurrents, depending on the photoelectrode potential. This switching is called the PEPS effect (photoelectrochemical photocurrent switching).

Anodic and cathodic photocurrent generation depends on the oxidation state of the surface species: the potential at which the photocurrent direction changes correlates very well with redox potential of the complex chemisorbed at titanium dioxide surface. At potentials more positive as compared to $E_{1 / 2}$ of the surface complex the excitation of the surface species does not take place and therefore no electron injection into the conduction band may occur. Under such conditions only excitation of the semiconductor matrix may operate resulting in anodic photocurrent generation (Fig. 2b). This behavior is identical to that of an unmodified n-type semiconductor, for which anodic photocurrents can be observed (Fig. 2a).

Reduction of the surface complex opens a possibility of cathodic photocurrents generation (Fig. 2c). Excitation of the surface species gives an electron in the conduction band: this state corresponds to the excited states of the surface complex. Due to a significant negative polarization of the conducting support an electron transfer from the conduction band to the electrode is not favored. The reverse process is much more probable, i.e. the electron transfer from the electrode to the photochemically oxidized surface species (partially emptied surface states) and a concomitant reduction of the electron acceptor in the electrolyte resulting in the cathodic photocurrent generation. A good candidate for the electron acceptor

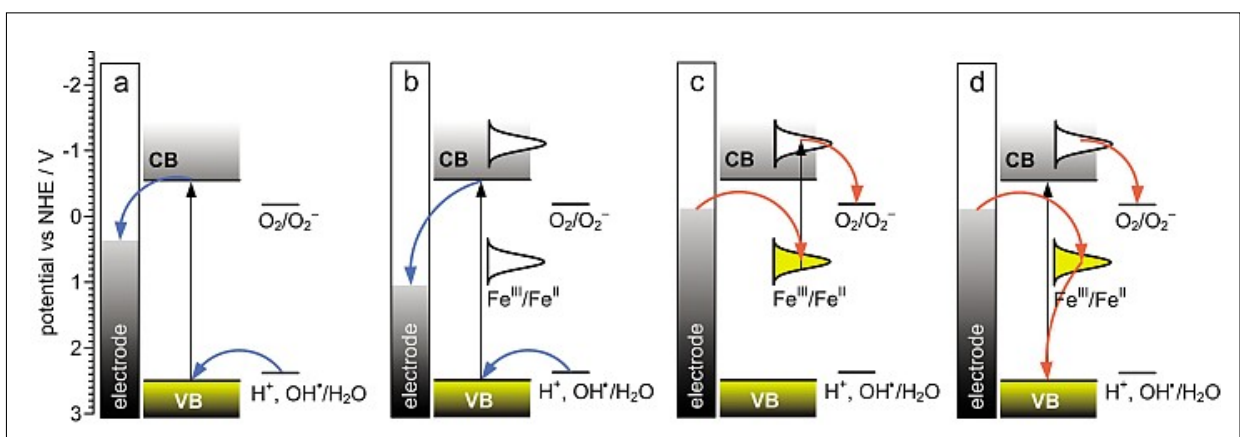

Fig. 2. The simplified mechanism of photocurrent generation at neat $\mathrm{TiO}_{2}$ photoelectrode $(\mathrm{a}), \mathrm{TiO}_{2}$ electrode with oxidized surface complex (b) and reduced surface complex (c, d)

is an oxygen molecule $\left(E_{\mathrm{O} 2 / \mathrm{O} 2^{-}}=-0.16 \mathrm{~V}\right.$ $v s$. NHE). ${ }^{[38,39]}$ Excitation of the modified material within the semiconductor absorption also results in cathodic photocurrent. The hole in the valence band is neutralized with an electron from the surface species, which are in turn reduced electrochemically (Fig. 2d).

This simple mechanism was confirmed by quantum chemical calculations. A series of model complexes containing an iron center covalently linked to the $\left[\mathrm{Ti}(\mathrm{OH})_{3}\right]^{+}$ moiety was designed. Density functional theory calculations showed that in all studied cases the HOMO orbital is localized on the surface complex (partially delocalized between iron center and ligands) while LUMO is exclusively localized on titanium(IV) center (Fig. 3). Furthermore, the electronic transition between these frontier orbitals is allowed and the calculated energy of this transition corresponds well to the low energy tail of the reflectance spectra of modified $\mathrm{TiO}_{2} \cdot{ }^{[37]}$

The formation of space charge layer also contributed to the photocurrent switching process. Depending on the nature of the surface species (charge and electron donor/ acceptor character) accumulation, depletion or inversion layers can be formed. ${ }^{[40-42]}$ In the absence of surface species the semiconductor crystals contain a uniform distribution of charge (Fig. 4a). Deposition of oxidized species, acting as electron acceptors, onto the semiconductor surface increases the majority carrier concentration close to the surface of the crystal. The space charge layer thus formed is called an accumulation layer. The bands of the semiconductor bend downwards owing to decreased potential energy of electrons approaching the surface (Fig. 4b). Accumulation of negatively charged molecules (or strong electron donors) onto the semiconductor surface causes lowering of the charge carrier concentration close to the surface. The space charge layer thus formed is the depletion layer (Fig. 4c). When the depletion layer extends deep into the semiconductor crystal the Fermi level near the surface may fall below the intrinsic level (half way between the valence and conduction bands). In this case the surface part of the semiconductor appears to behave like a p-type semiconductor, while the inner part of the crystal retains the n-type character (Fig. 4d).

Depending on the subtle interplay between the electronic (Fig. 2) and electrostatic (Fig. 3) factors influencing the photocurrent switching phenomena two main types of switching processes can be observed. In the simplest case a material with oxidized surface complex generates an anodic photocurrent within its whole absorption spectrum. Upon reduction of the surface species cathodic photocurrents evolve within the whole absorption spectrum. As the reduc- 


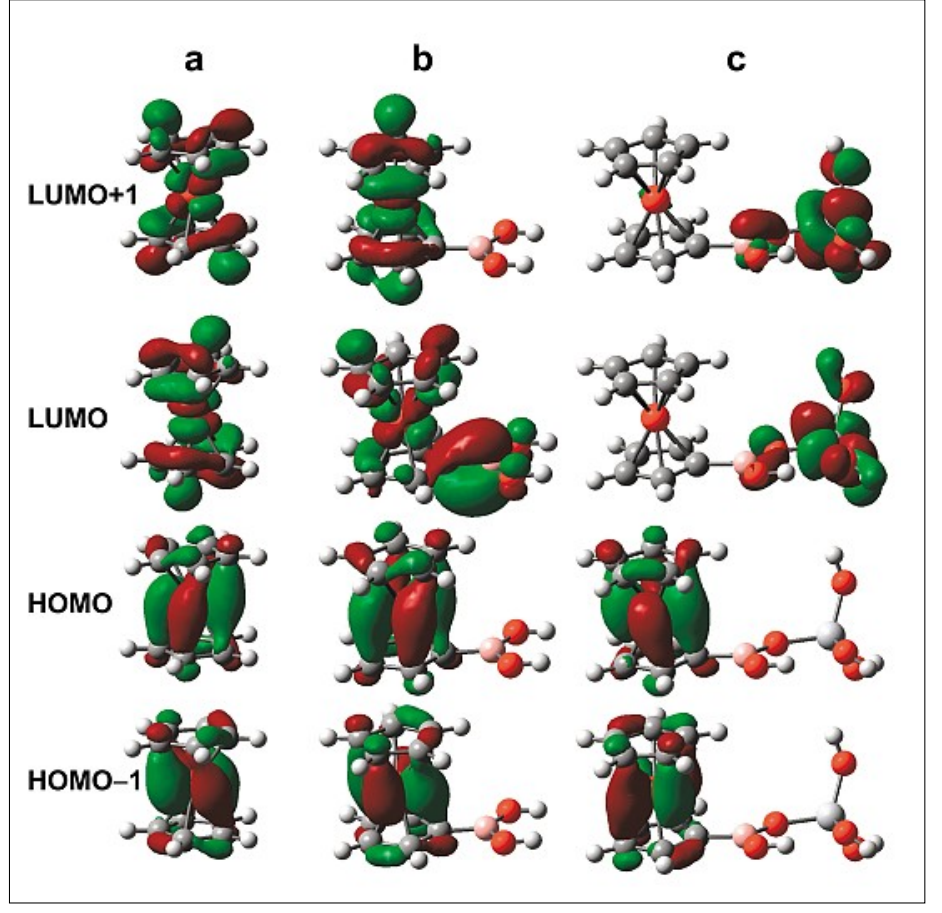

(a)

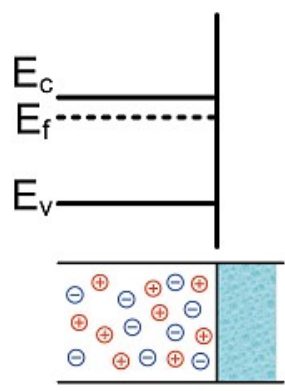

(b)

(c)

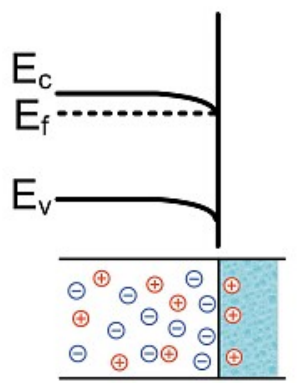

Fig. 3. Frontier molecular orbitals of (a) ferrocene, (b) ferrocenylboronic acid and (c) ferrocenylboronatetrihydroxytitanium(IV) model complex as calculated at the B3PW91/6$311++(d, p)$ level of theory by using tight convergence criteria

Fig. 4. Simplified electronic structures of semiconductor-electrolyte interfaces: (a) neat semiconductor; (b) semiconductor modified with oxidized form of the complex, accumulation layer is formed due to interaction with the surface complex; (c) semiconductor modified with partially reduced form of the complex, depletion layer is formed due to an interaction with the surface complex; (d) inversion layer is formed upon complete reduction of the surface species. In the case of small particles vertical shifts of VB and $\mathrm{CB}$ instead of band bending should be considered

\section{(a)}

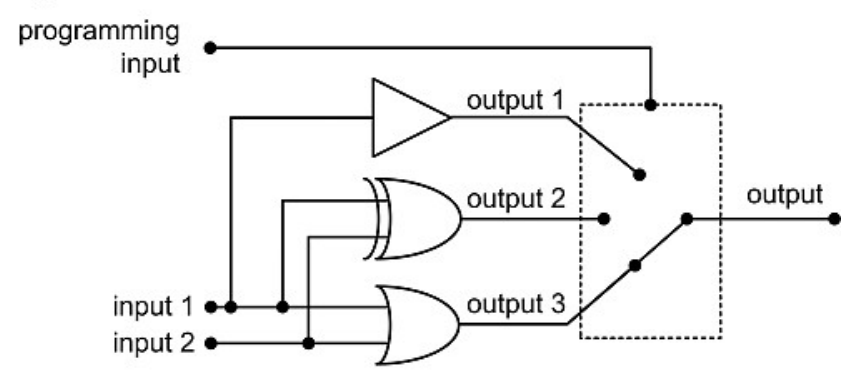

(b)

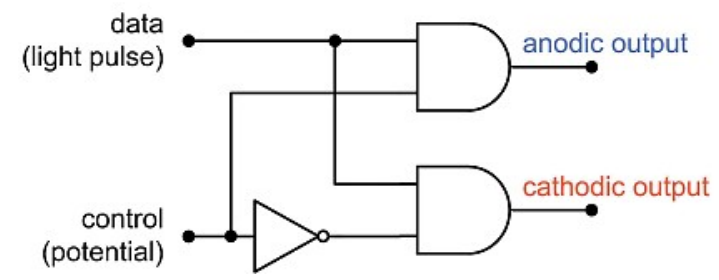

tion is usually associated with formation of the MPCT transitions, an efficient photosensitization within visible light is usually observed.

In some cases, however, photocurrent polarity depends on irradiation wavelength. This is the case for hexacyanoferrate-modified titanium dioxide, Prussian blue-modified cadmium sulfide and some other systems. At potentials corresponding to partial reduction/oxidation of the surface species it is possible to excite selectively the inner part of semiconductor particles or the surface complex exclusively. This leads to generation of anodic or cathodic photocurrents, respectively, depending on the incident light wavelength. At these potentials bichromatic illumination (e.g. from two LEDs) results in a null net photocurrent. ${ }^{[33,36]}$

Semiconducting materials which show any photocurrent switching mode can serve as a platform for construction of optoelectronic logic gates and other switching devices. The operation mode of these devices depends on the switching characteristics of the surface-modified semiconductor. Two functional examples of devices based on $\mathrm{TiO}_{2}$ modified with hexacyanoferrate $\left(\mathrm{HCF} @ \mathrm{TiO}_{2}\right)$ and Prussian blue (PB@ $\mathrm{TiO}_{2}$ ) are given below.

At potentials ensuring a complete oxidation of the surface species (e.g. +400 $\mathrm{mV}$ vs. $\mathrm{Ag} / \mathrm{AgCl}$ ) pulsed irradiation of the $\mathrm{HCF} @ \mathrm{TiO}_{2}$ material with violet light (LED $\lambda_{\text {max }}=400 \mathrm{~nm}$ ) results in anodic photocurrent pulses, while irradiation with blue light $\left(\right.$ LED $\lambda_{\max }=460 \mathrm{~nm}$ ) does not generate any photocurrent. Simultaneous irradiation with two LEDs yields the same effect as the violet diode alone (Fig. 5a, output 1). Electrochemical reduction of the surface species is achieved at lower potentials. Excitation of this material with violet and blue diodes leads to generation of cathodic photocurrent. Simultaneous irradiation by both diodes results in higher intensity of the photocurrent. Such behavior of the photoelectrode at $-200 \mathrm{mV}$ vs. $\mathrm{Ag} / \mathrm{AgCl}$ corresponds to the OR logic gate (Fig. 5a, output 3).[33,36]

Much more interesting photocurrent profiles were recorded upon partial oxidation of the surface complex. ${ }^{[33,36]}$ Irradiation with $400 \mathrm{~nm}$ light results in generation of anodic photocurrent which is consistent with excitation of the inner part of semiconductor particles. Irradiation with 460 $\mathrm{nm}$ light results in generation of cathodic photocurrent. Simultaneous irradiation with two diodes gives a null net photocurrent as anodic and cathodic photocurrents compensate effectively (Fig. 5a, output 2). This behavior analyzed in terms of Boolean logic corresponds to the XOR gate. The photocurrent compensation, however, requires adjustment of light intensity of both sources (and/or a subtle adjustment of
Fig. 5. Electronic equivalent circuits of logic devices based on nanocomposite photoelectrodes: a reconfigurable OR/ $\mathrm{XOR} / \mathrm{YES}$ logic gate (a) and a two channel optoelectronic demultiplexer (b) 
the photoelectrode potential) such that absolute values of anodic and cathodic photocurrents are equal.

The logic device based on semiconducting photoelectrodes shows another important feature. It should be noted that the logic analysis of this systems can be much more complex if the photoelectrode potential is considered as the third input channel. In this context the logic system described here is a chemical model of a reconfigurable logic device, function of which can be programmed by the user. ${ }^{[43,44]}$ Changes in applied potential can switch the logic behavior of the photoelectrode between three different regimes:

i) transmission of the input 1 signal to the output neglecting input 2 (YES),

ii) computation of the $\mathrm{XOR}$ function of two input values and

iii) computation of the OR function of two input values (Fig. 5a).

Another switching behavior is observed for the PB@TiO, material: photocurrent switching is virtually wavelength-independent. ${ }^{[34]}$ At potentials higher than the PB redox potential the electrode generates anodic photocurrents, while at lower potentials cathodic photocurrents are observed. One can assign the logic values of ' 0 ' and ' 1 ' to the negative and the positive polarization of the photoelectrode, respectively. The switching characteristics allows it to be used as an optoelectronic two channel demultiplexer (Fig. 5b). This device collects information in form of light pulses and converts it into photocurrent pulses. Furthermore, the sign (direction) of photocurrent pulses depends on the photoelectrode potential. Other words, information in form of photocurrent pulses can be directed into two output channels: cathodic or anodic. An electronic equivalent of this logic device is composed of two AND and one NOT logic gates. The input data signal (light pulses) is applied to one input of both AND gates, the control signal goes to one AND gate directly while to the other via an inverter. In this configuration one of the AND gates is in the ON and the other is the OFF state.

The chemical systems described above are simple functional models of logic devices. Information is supplied to the system by means of light pulses, and processed information is retrieved in form of current pulses. This behavior is quite unique for chemical logic systems and allows a facile communication between various electronic silicon-based devices and chemical logic system. Furthermore, application of photoelectrochemical processes mitigates the problems of concatenation and interfacing: both input and output signal of these molecular devices can be easily 'understood' by classical electronic devices.

\section{Acknowledgements}

Authors thank Ms. Bela Szaciłowska for editorial support during the preparation of the manuscript. Financial support from the Polish Ministry of Science and Higher Education (grants No. PB0941/T08/2005/28 and PBZ$\mathrm{KBN}-118 / \mathrm{T} 09 / 8)$ is gratefully acknowledged.

Received: October 29, 2007

[1] J. D. Meindl, Q. Chen, J. A. Davis, Science 2001, 293, 2044.

[2] A. P. de Silva, B. McCaughan, B. O. F. McKinney, M. Querol, Dalton Trans. 2003, 1902.

[3] A. P. de Silva, N. D. McClenaghan, Chem. Eur. J. 2004, 10, 574.

[4] J. F. Callan, A. P. de Silva, D. C. Magria, Tetrahedron 2005, 61, 8551.

[5] K. P. Zauner, Crit. Rev. Solid State Mat Sci. 2005, 30, 33 .

[6] V. Balzani, A. Credi, M. Venturi, ChemPhysChem 2003, 3, 49.

[7] V. Balzani, A. Credi, M. Venturi, Chem. Eur. J. 2002, 8, 5525 .

[8] V. Balzani, M. Venturi, A. Credi, 'Molecular Devices and Machines - A Journey into Nanoworld', Wiley-VCH, Weinheim, 2003.

[9] K. Szaciłowski, Z. Stasicka, Coord. Chem. Rev. 2002, 229, 17.

[10] D. C. Magri, T. P. Vance, A. P. de Silva, Inorg. Chim. Acta 2007, 360, 751.

[11] A. P. de Silva, Y. Leydet, C. Lincheneau, N. D. McClenaghan, J. Phys.: Condens. Matter 2006, 18, S1847.

[12] F. M. Raymo, S. Giordani, Proc. Natl. Acad. Sci. USA 2002, 99, 4941.

[13] V. Balzani, A. Credi, F. R. Raymo, J. F. Stoddart, Angew. Chem., Int. Ed. 2000, 39, 3348.

[14] F. R. Raymo, Adv. Mater. 2002, 14, 401.

[15] F. R. Raymo, M. Tomasulo, Chem. Eur. J. 2006, 12, 3186.

[16] J. Wu, W. Pisula, K. Müllen, Chem. Rev. 2007, 107, 718

[17] K. Walzer, B. Maennig, M. Pfeiffer, K. Leo, Chem. Rev. 2007, 107, 1233

[18] E. Menard, M. A. Meitl, Y. Sun, J.-U. Park, D. J.-L. Shir, Y.-S. Nam, S. Jeon, J. A. Rogers, Chem. Rev. 2007, 107, 1117.

[19] A. R. Murphy, J. M. J. Frechét, Chem. Rev. 2007, 107, 1066

[20] T. P. I. Saragi, T. Spehr, A. Siebert, T. Fuhrmann-Lieker, J. Salbeck, Chem. Rev. 2007, 107, 1101.
[21] M. M. Ling, Z. Bao, Chem. Mater. 2004 16, 4824.

[22] M. Bendikov, F. Wudl, D. F. Perepichka, Chem. Rev. 2004, 104, 4891.

[23] C. R. Newman, C. D. Frisbie, D. A. da Silva Filho, J.-L. Brédas, P. C. Ewbank, K. R. Mann, Chem. Mater. 2004, 16, 4436.

[24] J. C. Love, L. A. Estroff, J. K. Kriebel, R. G. Nuzzo, G. M. Whitesides, Chem. Rev. 2005, 105, 1103.

[25] F. J. M. Hoeben, P. Jonkheijm, E. W. Meijer, A. P. H. J. Schenning, Chem. Rev 2005, 105, 1491.

[26] J.-H. Fuhrhop, T. Wang, Chem. Rev. 2004 104, 2901.

[27] K. Rajeshwar, N. R. de Tacconi, C. R. Chenthamarakshan, Chem. Mater. 2001, 13, 2765.

[28] H. N. Gosh, J. B. Ashbury, Y. Weng, T. Lian, J. Phys. Chem. B 1998, 102, 10208.

[29] F. De Angelis, A. Tilocca, A. Selloni, J. Am. Chem. Soc. 2004, 126, 15024.

[30] W. Macyk, G. Stochel, K. Szaciłowski, Chem. Eur. J. 2007, 13, 5676.

[31] C. D. Lindstrom, X.-Y. Zhu, Chem. Rev. 2006, 106,4281 .

[32] M. Hebda, G. Stochel, K. Szaciłowski, W. Macyk, J. Phys. Chem. B 2006, 110, 15275 .

[33] K. Szaciłowski, W. Macyk, G. Stochel, J. Am. Chem. Soc. 2006, 128, 4550.

[34] K. Szaciłowski, W. Macyk, G. Stochel, J. Mater. Chem. 2006, 16, 4603.

[35] K. Szaciłowski, W. Macyk, Comp. Rend. Chimie 2006, 9, 315.

[36] K. Szaciłowski, W. Macyk, Solid State Electron. 2006, 50, 1649.

[37] K. Szaciłowski, W. Macyk, M. Hebda, G. Stochel, ChemPhysChem 2006, 7, 2384.

[38] D. T. Sawyer, J. S. Valentine, Acc. Chem. Res. 1981, 14, 393.

[39] D. M. Stanbury, Adv. Inorg. Chem. 1989, 33,69 .

[40] C. A. Koval, J. N. Howard, Chem. Rev. 1992, 92, 411 .

[41] A. Hagfeldt, M. Grätzel, Chem. Rev. 1995, 95,49 .

[42] A. L. Linsebigler, G. Lu, J. T. Yates Jr., Chem. Rev. 1995, 95, 735.

[43] J. Becker, R. J. Hartenstein, Systems Arch. 2003, 49, 127.

[44] C. P. Collier, E. W. Wong, M. Belohradsky, F. M. Raymo, J. F. Stoddart, P. J. Kuekes, R. S. W. R. Heath, Science 1999, 285, 391. 